\title{
A formação de profissionais para a atenção integral à saúde do idoso: a experiência interdisciplinar do NAI - UNATI/UERJ
}

\author{
Training professionals for delivering ingreated health care \\ to the aged: the interdisciplinary experience of NAI - UNATI/UERJ
}

Luciana Branco da M otta ${ }^{1}$

Célia Pereira Caldas ${ }^{2}$

M ônica de Assis ${ }^{1}$

\footnotetext{
${ }^{1}$ Núcleo deAtenção ao Idoso, UnATI - HUPE/ UERJ. R. São Francisco Xavier $524,10^{\circ}$ andar, bloco F, sala 10147, M aracanã. 20550-013 Rio de Janeiro RJ. lumotta@uerj.br ${ }^{2}$ Faculdade deEnfermagem, UnATI/UERJ.
}

Abstract Thetraining of professionals in thefield of healthcare for theaged is one of the priorities of the national policy for the aged in Brazil due to the accelerated aging of the population. The Núcleo deAtenção ao I doso (NAI), a unit of the 0 pen University of theThird Age/UERJ (UNATI/UERJ) develops an educational program in this field, based on practical care delivery with emphasis to inter-disci plinarity and teamwork. The program includes different training levels and modalities: Residency, Specialization, Professional Practice and Graduation. The program includes an introductory course in gerontology and geriatrics common to all areas, and specific theoretical-practical qualification coordinated by the professional staff from the respective areas. The practical activities occur in different sceneries: long term care institutions, health promotion educational settings, outpatient facilities and the university hospital. Interdisciplinary thinking and acting is a continuous exercise, and the team should be open to innovative strategies. The experience is a contribution to the increasing social demand for qualified professionals committed with the principles of the U nified H ealth System and integrated health care.

Key words Professional qualification, Human aging, Elderly health, Integrated attention, Interdisciplinarity
Resumo A capacitação deprofissionais para atuar na área de envelhecimento e saúde do idoso éuma das ações prioritárias da política nacional do idoso no Brasil, em função do acelerado envelhecimento populacional do país. $\mathrm{O} \mathrm{N}$ úcleo deAtenção ao I doso, serviço do H ospital U niversitário Pedro Ernesto, vinculado à U niversidade Aberta da Terceira I dade/UERJ, desenvolve programa de ensino nesta área, a partir da experiência assistencial, tendo como eixo a integralidade da atenção e 0 trabalho interdisciplinar. 0 programa inclui modalidades de ensino em nível de residência, especialização, treinamento profissional e estágio de graduação. A programação teórica inclui um curso de Introdução à Saúde do I doso, comum às diversas áreas profissionais. A capacitação teórica e prática específica é coordenada pelos preceptores das respectivas áreas. As atividades práticas ocorrem em diferentes cenários, incluindo 0 acolhimento, a promoção da saúde, a atenção ambulatorial, a hospitalar e a de longa permanência. A interdisciplinaridade éum exercício contínuo que supõe abertura a estratégias inovadoras. A experiência representa uma contribuição à demanda social crescente de capacitação profissional em um modelo de atenção comprometido com princípios do SU S e com o cuidado integral.

Palavras-chave Capacitação profissional, EnveIhecimento, Saúde do idoso, A tenção integral, Interdisciplinaridade 
Introdução

A implantação de políticas e programas considerando o novo perfil demográfico do país inclui a necessidade de ampliação quantitativa e qualitativa de profissionais para atuar na área do enve Ihecimento. Esta necessidadetem sido destacada na Política Nacional do Idoso ${ }^{1,2}$, e mais recentemente na Política Nacional de Saúde do Idoso ${ }^{3}$, que apontam a importância da criação de disciplinas em Geriatria e Gerontologia nos cursos de graduação e de núcleos de formação profissional em nível de pós-graduação.

Tendo em vista estas diretrizes políticas, a UnATI (Universidade Aberta da Terceira Idade) da UERJ (Universidade do Estado do Rio de Janeiro) vem implementando e expandindo atividades de ensino voltadas à formação e capacitação de recursos humanos em Geriatria e Gerontologia. Com o acúmulo de experiência em assistência e pesquisa em saúde do idoso, várias modalidades detreinamento, em nível degraduação e pós-graduação, têm se consolidado no âmbito do programa de atenção integral desenvolvido.

O NAI (N úcleo de Atenção ao Idoso) é um serviço de saúde do H ospital Universitário Pedro Ernesto (HUPE), vinculado à UnATI, cujo objetivo é a atenção integral à saúde do idoso. Este serviço conta com uma equipe formada por profissionais de diferentes áreas, numa perspectiva de ação integrada e interdisciplinar. O NAI de senvolve diversas modalidades de ações que visam à atenção à saúde do idoso eà disponi bilização de cenários variados para o treinamento.

A construção do projeto pedagógico e do conteúdo programático do treinamento interdisciplinar desenvolvido no NAI vem acontecendo a partir da experiência prática. A fundamentação teórica adotada emerge dos desafios encontrados na realidade, uma vez que o envel hecimento populacional brasileiro aponta para a necessidade de capacitação de profissionais aptos a lidar com as especificidades da atenção ao idoso em face de demandas sociais crescentes em diversas áreas, como as de prestação de serviço, pesquisa, políticas públicas, entre outras.

0 foco do presente artigo é descrever o programa de ensino desenvolvido neste serviço de saúde, quese inserenuma instituição voltada para o idoso, com uma experiência de quinze anos. Pretende-se com isso contribuir para uma meIhor compreensão da lógica da formação profissional voltada para a promoção da saúde do idoso e compartilhar com o público leitor os marcos de um modelo de capacitação quetem como eixos a integralidade, a interdisciplinaridade e 0 cuidado.

\section{A formação profissional \\ para a aten ção à saúde do idoso}

A formação do profissional de saúde que atua na atenção ao idoso deve ter como base o perfil do gerontólogo, que éapontado por M artins Sá ${ }^{4}$ como aquele apto a: apreender, histórica e criticamente, o processo do envelhecimento em seu conjunto; compreender o significado social da ação gerontológica; situar o desenvolvimento da gerontologia no contexto sócio-histórico; atuar nas expressões da questão da velhice e do enveIhecimento, formulando e implementando propostas para o enfrentamento; realizar pesquisas que subsidiem a formulação de ações gerontológicas; compreender a natureza interdisciplinar da gerontologia buscando ações compatíveis no ensino, pesquisa e assistência; zelar por uma postura ética esolidária no desempenho de suas funções; orientar a população idosa na identificação de recursos para 0 atendimento às necessidades básicas e de defesa de seus direitos. Este perfil é baseado nos objetivos da educação gerontológi$\mathrm{ca}$, que podem ser sintetizados em formar recursos humanos capazes de compreender o "ser veIho" e o processo de envelhecimento em suas dimensões conceituais, sociais, políticas, profissionais e éticas eformular eimplementar propostas para o enfrentamento das questões gerontológicas na sociedade contemporânea de modo eficaz, eficientee efetivo.

Podemos dizer que o currículo, entendido como relacionado às formas de organização do conhecimento, é um "artefato social e cultural". O currículo não é "transcendente e atemporal ele tem uma história, vinculada a formas específicas e contingentes de organização da sociedade e da educação" ${ }^{\prime \prime}$. Segundo M oreira ${ }^{5}$, os estudos sobre currículo abordam três temas centrais: 1) a ideologia, isto é, como a classe dominantetransmite suas idéias sobre o mundo social visando a reprodução da estrutura social; 2) a cultura, onde o currículo éuma forma de transmissão cultural incorporando a significação ativa dos materiais recebidos, ou seja, a elaboração por partedo educando do que é transmitido. Está vinculado à estrutura de grupos ou classes sociais, sendo terreno de luta pela manutenção ou superação das divisões sociais; e, 3) o poder; o currículo veicula os interesses dos grupos e classes dominantes numa dada sociedade. 
Ao abordar o currículo como produto social e historicamente situado, busca-se refletir sobre a valorização da inclusão de conteúdos e do treinamento em Geriatria e Gerontologia. A pesar dos dados sobre o envelhecimento serem cada vez mais discutidos e da legislação existente, ainda não está clara ou devidamente valorizada a importância destes conteúdos para a sociedade e para o idoso como ator social.

Por competência, entendemos um termo polissêmico descrito como capacidade para aplicar habilidades, conhecimentos e atitudes; habilidade de utilizar o conhecimento a fim de chegar a um propósito; capacidade de utilizar conhecimentos ehabilidades adquiridos para o exercício profissional; capacidade de mobilizar saberes - saber-fazer, saber-ser, saber-agir; capacidade para resolução de um problema ${ }^{6}$.

Quanto a capacidade de mobilização de conhecimentos: "só há competência estabilizada quando a mobilização dos conhecimentos supera o tatear reflexivo ao alcance de cada um e aciona esquemas constituídos"7. Segundo Bastien, citado por Perrenould ${ }^{7}$, um especialista écompetente porque é capaz de dominar rapidamente situações comuns com esquemas que entram em ação automaticamente e porque écapaz de, após uma reflexão, coordenar e diferenciar rapidamente os esquemas de ação e os conhecimentos para enfrentamento de situações inéditas.

Para DeLuiz ${ }^{8}$, a mudança para um novo modelo de organização do trabalho baseado em competências não mais enfatiza o saber escolar ou técnico-profissional, mas a capacidade de mobilizar saberes a fim de resolver problemas ou imprevistos, reconfigurando a importância atribuída às qualificações sociais e à subjetividade do indivíduo. Assim, ao buscar o sentido da competência na atuação profissional junto ao idoso, objetiva-se não só o conhecimento, mas sua contextualização dentro do processo de envelhecimento e da prestação de serviços, a capacidade deatuação frenteà imprevisibilidade ediversidade de situações, incluindo o trabalho em equipe interdisciplinar e a mobilização de conteúdos diversos buscando a atuação integral.

0 trabalho em saúde se caracteriza por ser um trabalho reflexivo, que depende da colaboração de saberes distintos como o científico, 0 técnico, os sociais e os provenientes de dimensões éticas e políticas. É um trabalho marcado também pela complexidade, isto é, a diversidade profissional, dos atores, das tecnologias, das relações sociais e interpessoais, da organização do espaço e dinâmica. Outras características do tra- balho em saúde são: a heterogeneidade devido à variedade de processos de trabal hos coexistentes e; a fragmentação conceitual, do pensar e fazer, da técnica (pluralidade profissional) e social (divisão social do trabalho e entre as categorias). Por fim, o trabalho em saúde é marcado pelas relações interpessoais entre os profissionais da equipe, eentreestes e o usuário deforma bastante significativa. Isto envolve mudanças importantes para a formação profissional em saúde ${ }^{8}$.

Assim, competência é "[...] mecanismo subjacente que permite a integração de múltiplos conhecimentos e atos necessários à realização da ação" ${ }^{\prime \prime}$, sendo condição de desempenho, pois expressa os recursos articulados emobilizados frente a uma situação. 0 desempenho é conseqüência das competências por ser dependente destas; porém, uma única ação pode mobilizar várias competências, que não correspondem apenas ao resultado do trabalho, mas à reflexão das condições da produção, meios usados, finalidade e organização da ação, além das condiç̧̃̃es subjetivas e sociais. A formação baseada em competências se denominou pedagogia diferenciada, e se fundamenta em um processo centrado na aprendizagem e não no ensino, na valorização do aluno como sujeito e na construção do conhecimento?.

A formação de recursos humanos na área de saúde do idoso está vinculada a uma compreensão de processo de envel hecimento e suas repercussões biopsicossociais, em face da qual seimpõe a necessidade do trabalho interdisciplinar à luz de novos paradigmas sobre saúde-doença, papel e objetivos do profissional de saúde. Estes pressupostos importam no conteúdo ehabilidades a serem desenvolvidos durante o treinamento.

\section{A proposta do Núcleo de Atenção ao I doso}

O Núcleo de Atenção ao Idoso foi criado como ambulatório de geriatria do HUPE, em 1990, com base em princípios da integralidade da atenção à saúde e do trabalho em equipe interdisciplinar, tendo como objetivos a formação de recursos humanos e o desenvolvimento de políticas e de novas metodologias no trabalho em saúde com a população idosa.

Instalado desde 1996 no espaço físico da UnATI, o NAI foi ampliando suas atividades, centradas inicialmentena atenção ambulatorial denível secundário. Atualmente, o NAI mantém-secomo unidade especializada no atendimento a idosos com dependência e/ou maior risco defragilização 
e desenvolve projetos específicos em neurogeriatria, urogeriatria e otimização cognitiva. Além da assistência ambulatorial, o NAI desenvolve ações no âmbito da atenção hospitalar em enfermarias declínica médica do HU PE; da atenção asilar, através deparceria com instituição filantrópica delonga permanência; e da promoção de saúde, através deações educativas e preventivas abertas aos idosos e ao público em geral.

A equipe do NAl é composta por profissionais de medicina, enfermagem, serviço social, nutrição, fisioterapia, psicologia, fonoaudiologia e odontologia. A lógica de trabal ho é pautada na busca de construção interdisciplinar, segundo uma matriz transversal baseada no desenvolvimento dos diversos projetos. Cada projeto tem um ou dois coordenadores que são responsáveis por sua organização e pela articulação da equipe participante, composta por profissionais e treinandos das várias áreas. A matriz por área profissional é vertical e permite a interlocução com os serviços pares no hospital universitário, garantindo o espaço de supervisão e ensino por área de conhecimento específico (Figura 1).

0 trabalho em equipe interdisciplinar éa estratégia central na busca da integralidade da atenção, tema rico em conotações e de extrema atualidade no debate sobre o direito à saúde no Brasil $^{10}$. Na experiência do NAI, integralidade pode ser entendida como busca de apreensão ampliada das necessidades de saúde, que considere o idoso e suas famílias como sujeitos, em seu con- texto histórico-social, numa interação que possibilite a construção compartilhada de respostas assistenciais ea otimização do cuidado. Ao mesmo tempo, concebe-se a assistência também como espaço que deve se inserir politicamente, ampliando o espaço de informação, debate e ações no sentido de viabilização dos direitos sociais dos idosos.

A interdisciplinaridade é intrínseca ao processo de produção e aplicação do conhecimento da Gerontologia, pela necessária integração de múltiplos aspectos na compreensão do envelhecimento. 0 debate sobre interdisciplinaridade surge como crítica à fragmentação do saber e da produção de conhecimento. 0 reconhecimento da realidade como complexidade organizada implica que se busque compreendê-la mediante estratégias dinâmicas e flexíveis de organização da diversidade percebida, de modo a se compreender as múltiplas interconexões nela existentes ${ }^{11}$.

Feuerwerker ${ }^{12}$ afirma que no campo da saúde a interdisciplinaridade acena com a possi bilidade da compreensão integral do ser humano e do processo saúde-doença. Para Campos ${ }^{13}$, os conceitos de núcleo de saber e campo de saber evidenciam esta relação entre o que é específico de cada área profissional e o que se constrói a partir da interface entre as áreas e da confluência de objetivos. Núcleo de saber, para o autor, refere-se ao conjunto de saberes e responsabilidades específicos de cada profissão ou especialidade, marcando assi $m$ os elementos de singularidade que de-

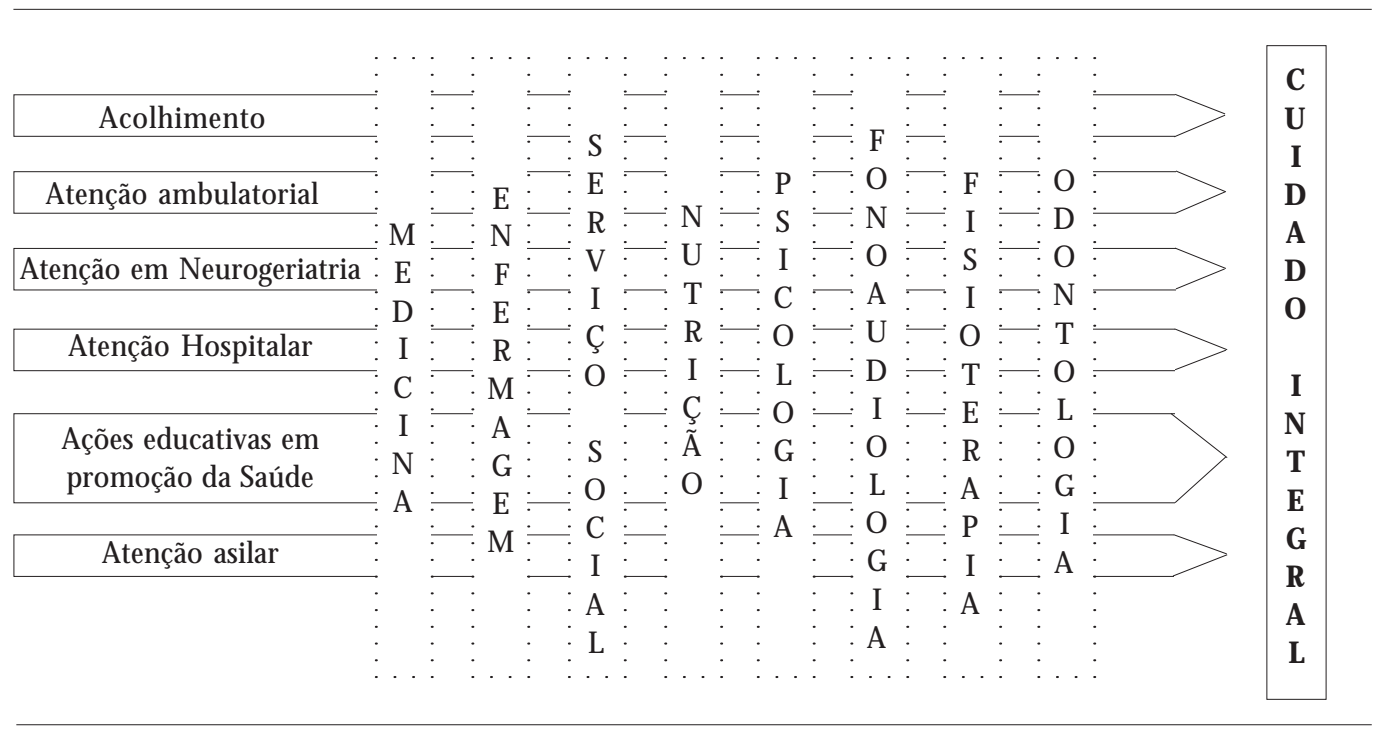

Figura 1. Organização do processo de trabalho do Núcleo de Atenção ao Idoso. 
finem cada profissional ou especialista, conhecimentos eações de competência decada profissional ou especialidade. Campo de saber, por sua vez, são as "competências e responsabilidades confluentes a várias profissões ou especialidades" . No trabalho em equipe, o campo de saber tende a se alargar, possibilitando a emergência de novas práticas, geradas no exercício de troca entre os saberes e da busca de respostas mais abrangentes e efetivas por parte dos profissionais.

Segundo Vasconcelos ${ }^{14}$, o exercício interdisciplinar em contexto de trabalho cooperativo e de horizontalização das relações de poder tende à constituição de uma área de saber com autonomia teórica e operativa própria, baseada em uma "axiomática geral compartilhada" ou conjunto devalores e princípios comuns que orienta as ações da equipe.

É na perspectiva da construção do cuidado integral, apoiada numa dinâmica de gestão participativa do Núcleo e da organização do trabalho por projetos transversais às áreas profissionais, queo N AI tem buscado al inhar as ações deensino.

\section{O programa de treinamento em saúde do idoso}

O programa possui atualmente as seguintes modalidades de treinamento: estágio supervisionado, residência em geriatria, treinamento profissional e treinamento prático para alunos do curso de especialização em Geriatria e Gerontologia da UnATI/UERJ.

A formação proposta baseia-se na inserção dos alunos na dinâmica geral da equipe e nos subprojetos do NAI. No plano assistencial, a linha mestra do treinamento prático éa reflexão e a elaboração conjunta do plano terapêutico para cada usuário do serviço. A participação do aluno nesta atividade permite ampliar sua compreensão sobre o papel de cada profissional e as interfaces necessárias na construção de alternativas às questões apresentadas.

0 trabalho em equipe é uma construção da prática diária. A WHO $\mathrm{O}^{15}$ aponta que a educação multiprofissional permiteao aluno aprender como interagir com os demais, não se superpondo ao currículo específico de cadaárea profissional, mas sendo complementar. 0 aprendizado se dá no contato direto com os usuários em diferentes cenários, tendo como objetivo comum dar respostas às questões de saúde da população atendida.

Para Garcia ${ }^{16}$, o cotidiano permite tornar a educação significativa, pois conjuga a vivência de situações ao processo do conhecimento, possibilitando o questionamento de práticas sociais e a instrumentalização para o conhecer e 0 agir.

0 processo pedagógico utilizado é o da Problematização ${ }^{17}$. Estese baseia no fato de que, frente a mudanças, o importante não são os conhecimentos, idéias ou comportamentos aprendidos, mas a capacidade do aluno, como participantee agente de transformação social, de detectar os problemas e buscar soluções. Assim, a capacidade a ser desenvolvida é a de fazer perguntas relevantes, possibilitando o entendimento da situação, e ser capaz de resolvê-las adequadamente. 0 processo "ensino-aprendizagem" relacionado a uma determinada realidade se inicia levando os alunos a observar a realidade em si. No segundo momento, os alunos identificam os pontos-chave do problema ou assunto eas variáveis mais determinantes da situação. No terceiro momento, os alunos passam à teorização do problema ao questionar as razões da realidade observada, buscando o entendimento deste. $\mathrm{Na}$ quarta fase, são formuladas hipóteses de solução para a questão, sendo então incentivada originalidade e a criatividade contrapondo-se com as limitações da própria realidade, a fim de se verificar a viabilidade e facti bilidade. $\mathrm{Na}$ última fase, as soluções mais viáveis e aplicáveis são colocadas em prática.

Estes paradigmas da educação e da atenção à saúde têm sido destacados pelo M inistério da Saúde e M inistério da Educação. Programas como o A prenderSU S e H umanizaSUS, a Educação Permanente em Saúde, nos últimos anos, exemplificam os caminhos quetêm sido referências para o NAI nas ações de ensino a partir da realidade assistencial. 0 programa vem sendo construído através de discussões da equipe e se organiza pela atividadee campo de saber comum, possibilitando o desenvolvimento de profissionais aptos a atuar, de forma inovadora, nas questões geradas pelo envelhecimento populacional em suas especificidades.

O programa de treinamento do NAI pretende oferecer instrumental teórico-prático na atenção à saúde do idoso para formar profissionais aptos a: atuar em serviços de saúde voltados para a população idosa enfatizando aspectos de promoção deautonomia eindependência dessegrupo etário; trabalhar em equipe interdisciplinar; propor plano terapêutico em equipe, objetivando a reabilitação, qualidade de vida e uso racional de recursos; trabalhar com as famílias e cuidadores como dimensão essencial da proposta assistencial. 


\section{Modalidades de treinamento}

O estágio supervisionado destina-se a estudantes de graduação da UERJ e/ou de outras universidades. Atualmente, o NAI oferece estágio nas áreas de Serviço Social, Fisioterapia, M edicina, Enfermagem e Nutrição. A carga horária varia de doze a vintehoras semanais e há possibilidade de bolsas nos projetos cadastrados como extensão universitária.

A residência éligada à Coordenadoria de De senvolvimento Acadêmico (CDA) do HUPE. Existem programas de residência em Geriatria e Ge rontologia (medicina) e em Saúde do Idoso em praticamente todas as áreas profissionais que compõem a equipe. 0 curso caracteriza-se como especialização (lato sensu) na modalidade detreinamento em serviço, e prevê carga horária de sessenta horas semanais. Os programas das áreas de saúde não médicas são reconhecidos pelos respectivos conselhos e atualmente passam por processo de reconhecimento como especialização junto ao M EC. A residência de psicologia já foi reconhecida como curso de especialização.

Os programas de residência têm formatos organizacionais variados e, em função disso, a inserção na área de saúde do idoso édiferenciada entre as áreas. A residência médica, credenciada em 1999, conta com três vagas no primeiro esegundo ano. A residência em Serviço Social, concentração em Saúde do Idoso, tem também duração de dois anos e conta atualmente com duas vagas. Os programas de residência em Psicologia e em Fisioterapia incluem o rodízio no primeiro ano e a concentração em Saúde do Idoso opcional no segundo ano. 0 programa de residência de Nutrição disponibiliza uma vaga para a Saúde do Idoso, sendo o primeiro ano voltado ao trabal ho junto à equipe no hospital e o segundo ano, às atividades ambulatoriais. A residência em Fonoaudiologia, devido ao escasso número de alunos, tem participação restrita à parte de carga horária no segundo ano e a residência de Enfermagem játeveinserção na forma de rodízio por breves períodos e passa por processo de re estruturação para garantir a concentração na área pelo período de dois anos.

A modalidade treinamento profissional em saúde do idoso foi criada em 2001 e configura-se como curso deaperfeiçoamento destinado a profissionais que demandem capacitação na área de atenção à saúde do idoso. A carga horária é de vinte horas semanais, incluindo atividades teóricas e práticas, por um período de seis meses a um ano. 0 pré-requisito é ter pelo menos dois anos de graduado eter disponibilidadedehorário para a programação prevista. A seleção é feita através de entrevista, com análise de currículo. Há uma ou duas vagas por cada área profissional.

Os alunos do curso de especialização em $\mathrm{Ge}$ riatria e Gerontologia da UnATI desenvolvem a Disciplina dePrática edeSeminário junto ao NAI . A Disciplina de Prática tem duração de doze semanas com a carga horária de doze horas semanais. Estas correspondem a três turnos de atendimento, com concentração nas atividades priorizadas pelos treinandos, conforme sua trajetória, interesses e disponibilidade dos projetos. A Disciplina de Seminário corresponde a doze encontros, nos quais são abordados temas relacionados ao desenvolvimento metodológico do trabalho no NAI, incluindo o treinamento prático na aplicação dos instrumentos mais utilizados para avaliar o idoso e na abordagem das principais síndromes geriátricas.

\section{Atividades teórico-práticas}

Em cada modalidade detreinamento, os treinandos se inserem em atividades teóricas e práticas comuns e específicas por área profissional.

As atividades teóricas comuns têm como base o curso de "Introdução à Saúde do Idoso", com carga horária de oitenta horas. 0 curso tem por objetivo apresentar aos alunos conceitos básicos degerontologia e saúde do idoso quefundamentam o trabalho na área. Ele inicia após a semana de recepção e ambientação dos treinandos e se divide nos seguintes módulos: Conceitos Básicos, Síndromes Geriátricas, Envelhecimento e Políticas Sociais, Psicologia do idoso: Aspectos sociais e clínicos, Promoção da Saúde, Processo de Reabilitação e Espaços de Atenção. As aulas têm duração de duas horas e ocorrem uma vez por semana.

Considerando a importância da capacidade funcional como indicador de saúde do idoso, na semana de recepção os alunos recebem orientação teórica e prática sobre avaliação funcional e cognitiva. São também apresentados e debatidos conceitos como trabal ho em equipe, interdisciplinaridade e planejamento terapêutico, na dinâmica do processo de trabalho em assistência eensino no NAl.

A partir de então, os treinandos passam a participar da reunião semanal de toda a equipe para supervisão das atividades assistenciais do ambulatório NAI e, conforme sua inserção, também das reuniões específicas de cada projeto. 
Estes espaços são prioritariamente de discussão da rotina do trabalho, porém incorporam discussões teóricas específicas, de acordo com as necessidades dos projetos e interesses da equipe.

As atividades teóricas específicas de cada área profissional são oferecidas de acordo com programação própria, sob responsabilidade de cada preceptor. Em geral, há supervisão e grupo de estudos periódicos, além da participação em módulos teóricos oferecidos pel os programas de residência. Tal prática preserva o espaço de reflexão e debate sob a ótica disciplinar, igualmente importante na qualificação profissional.

As atividades práticas comuns referem-se ao trabalho coletivo desenvolvido pela equipe, visando ao desenvolvimento dehabilidades como interdisciplinaridade, reconhecimento do papel do outro, comunicação com profissionais e usuários e construção partilhada de respostas assistenciais.

As atividades práticas comuns se caracterizam não apenas pelas atividades onde cada profissional atua e integra a sua ação a dos demais, através de interconsultas e reuniões de equipe, mas também pelas atividades efetivamente comuns, onde todos os treinandos realizam a mesma ação, resguardando eao mesmo tempo transpondo suas especificidades profissionais. É o caso do acolhimento dos idosos na porta-de-entrada do serviço (atendimento feito em dupla de profissionais a partir de um instrumento comum), e das ações educativas em saúde (realização degrupos e de atendimentos para avaliação multidimensional de qualidade de vida), espaços onde o trabal ho se caracteriza por objetivos e ações comuns às diversas áreas profissionais.

As atividades práticas específicas ocorrem em meio às atividades comuns a todos os treinandos, na própria dinâmica assistencial. Têm por objetivo a vivência prática e 0 amadurecimento dos alunos nas suas áreas de saber, articulando a elas conhecimentos mais estruturados sobre 0 processo deenvel hecimento. Estas atividades envolvem atendimento individual e em grupo aos idosos e suas famílias, conforme metodologia própria a cada área profissional.

Como forma de avaliação final, os residentes devem produzir uma monografia relacionada à experiência teórico-prática, com orientação deseu supervisor. Este trabalho deve ser apresentado ao final do período para a equipe do NAI, como forma de retroalimentar o processo ensinoaprendizado evalorizar a atividade acadêmica no programa de residência. $N$ as demais modalidades, a produção de trabalho final como estraté gia privilegiada para articulação teórico-prática étambém requerida, variando conformeo período de treinamento e o nível de aprendizado:

. Estágio supervisionado: relatório final de avaliação das atividades;

- Treinamento profissional: monografia ou elaboração escrita de caso clínico;

- Treinamento prático da especialização: elaboração de plano terapêutico interdisciplinar.

A elaboração do plano terapêutico interdisciplinar refere-se à própria experiência de discussão de casos oferecida no N AI nos diversos espaços de atenção e deve ser a estratégia para dar oportunidade ao aluno para demonstrar a sua capacidade de reconhecer de forma apropriada os problemas existentes e apontar suas possíveis alternativas ou soluções a partir da mobilização dos recursos da equipe.

\section{Avaliação do programa}

A avaliação do programa permite a retroalimentação do processo com vistas a ajustes contínuos e pode ser entendida como a possi bilidade de interpretar resultados eidentificar o nível alcançado a partir dos objetivos iniciais. A definição dos objetivos permite a escolha dos instrumentos a serem utilizados. Este processo visa à análise do desempenho do aluno e do curso pelos alunos ${ }^{18}$.

Cada modalidade de treinamento do programa tem uma forma de avaliação e é de responsabilidade do preceptor de cada área, conforme normas próprias. Porém, como há supervisão compartilhada com os responsáveis pelos projetos em que os treinandos estão inseridos, o consel ho de preceptores do N AI se reúne trimestralmente e avalia a inserção de cada aluno quanto ao conhecimento adquirido em gerontologia, à participação e 0 envolvimento no trabalho em equipe e às habilidades previstas para cada projeto de atuação. No momento de supervisão específica, as observações e sugestões feitas pelo Conselho são apresentadas a cada treinando, que participa do processo se auto-avaliando e avaliando o aprendizado no período correspondente.

Semestralmente, está programada uma reunião com treinandos e preceptores para avaliação do programa de treinamento. Os alunos são divididos em grupos multiprofissionais e orientados a avaliar cada área de atividade prática, com sugestões para os pontos considerados negativos. Esta avaliação éa base para as modificações no programa do período seguinte.

A avaliação dos alunos no curso "Introdução à Saúde do Idoso" éfeita através da presença eda 
elaboração de uma resenha de um dos textos de apoio, ao final decada módulo. É também solicitado ao aluno que avalie o módulo quanto ao conteúdo, professores, infra-estrutura e material de apoio, sendo aberto espaço para críticase sugestões. É requerida a presença mínima de $75 \%$ das aulas.

No período de 1997 a 2005, aproximadamente 171 alunos passaram pelo treinamento no NAI, nas diferentes modalidades. $O$ Quadro 1 especifica a partici pação de cada área profissional, por tipo de treinamento.

As avaliações dos alunos após os módulos e ao final de período têm sido positiva, com destaque para a organização do treinamento baseada nos projetos, o trabalho em equipe eo conteúdo teórico. De modo geral, as questões apontadas referem-se aos limites estruturais do trabalho, como a questão de recursos materiais e a pequena autonomia no projeto de atenção hospitalar pela não disponibilidade ainda da enfermaria do próprio NAI.

\section{Considerações finais}

0 programa de treinamento vem ao longo destes anos sendo discutido e remodelado buscando se adaptar às necessidades dos alunos e às especificidades de se trabalhar com o tema enveIhecimento.
Acreditamos que este seja um modelo de treinamento adequado a desenvolver competências necessárias para o profissional de saúde que trabalha com o envel hecimento e o usuário idoso, em um aprendizado ativo que permite a integração entreasáreas, deforma conjunta eresolutiva.

O foco na integralidade da atenção e no cuidado permitetrabalhar com objetivos como prevenção e promoção de saúde nos diversos níveis de atenção, a partir da compreensão ampliada do processo saúde-doença e do envelhecimento no curso de vida. É necessário que as oportunidades de formação nesta linha sejam multiplicadas para fazer face às demandas sociais crescentes pelo envelhecimento populacional, ao mesmo tempo contribuindo na construção de modelo assistencial pautado em princípios do SUS, tão necessário quanto ainda pouco consolidado em nosso contexto.

\section{Colaboradores}

LB M otta trabalhou na concepção, pesquisa bibliográfica e redação; CP Caldas trabalhou na concepção e revisão do texto; M Assis trabalhou na concepção e redação do texto.

Quadro 1. Número de alunos por área profissional e modalidade de treinamento, no período de 1997 a 2005 - NAI - UnATI / UERJ.

\begin{tabular}{|l|c|c|c|c|c|}
\hline \multirow{2}{*}{ Áreas profissionais } & \multicolumn{5}{|c|}{ Modalidades de treinamento } \\
\cline { 2 - 6 } & Residência & Treinamento profissional & Estágio & Especialização & Total \\
\hline M edicina & 17 & 8 & 4 & 18 & 47 \\
\hline Serviço Social & 9 & 6 & 8 & 2 & 25 \\
\hline Fisioterapia & 6 & 11 & & 18 & 35 \\
\hline Nutrição & 3 & 3 & 20 & 1 & 13 \\
\hline Enfermagem & 2 & 1 & 7 & 4 & 27 \\
\hline Psicologia & 14 & 8 & & 1 & 30 \\
\hline Fonoaudiologia & 4 & 5 & 2 & 3 & 12 \\
\hline Odontologia & & 1 & 47 & 50 & 6 \\
\hline Total & 55 & 43 & & 3 & 195 \\
\hline
\end{tabular}




\section{Referências}

1. Brasil. Lei $n^{\circ} 8.842$ de 4 de janeiro de 1994. Dispõe sobre a Política Nacional do Idoso, cria o ConseIho Nacional do Idoso e dá outras providências. Diário Oficial da União 1996; 5 jan.

2. Brasil. M inistério da Previdência e Assistência Social. Plano de Ação Governamental Integrado para o desenvolvimento da Política Nacional do Idoso. Brasília: Ministério da Previdência e Assistência Social/ Secretaria de Assistência Social; 1996.

3. Brasil. Portaria no $1395 / \mathrm{GM}$ de 9 de dezembro de 1999. Aprova a Política Nacional de Saúde do Idoso e dá outras providências. Diário Oficial da União 1999; 13 dez.

4. Martins de Sá JL. A formação de recursos humanos em Gerontologia: fundamentos epistemológicos e conceituais. In: Freitas EV, Py L, Cançado FAX, Gorzoni ML, organizadores. Tratado de geriatria e gerontologia. Rio de Janeiro: Guanabara Koogan; 2002. p. 1119-1124.

5. Moreira AFB, Silva TT. Sociologia e teoria crítica do currículo: uma introdução. In: Moreira AFB, Silva TT, organizadores. Currículo, cultura e sociedade. São Paulo: Cortez; 1994. p. 7-37.

6. Depresbiteris L. Certificação de competências: a necessidade de avançar numa perspectiva formativa. Formação. Humanizar cuidados de saúde: uma questão de competência 2001; 1(2):27-38.

7. Perrenould P. A noção de competência. In: Perrenould $P$, organizador. Construir as competências desde a escola. Porto Alegre: Artes M édicas; 1999. p.19-33.

8. DeLuiz N. Qualificação, competências e certificação: visão do mundo do trabalho. Formação. Humanizar cuidados de saúde: uma questão de compe tência 2001; 1(2):5-15.

9. Ramos MN. Qualificação, competências e certificação: visão educacional. Formação H umanizar cuidados de saúde: uma questão de competência 2001; $1(2): 17-26$.
10. M attos RA. Os sentidos da integralidade: algumas reflexões acerca de valores que merecem ser defendidos. In: Pinheiro RE, Mattos RA, organizadores. Os sentidos da integralidade na atenção e no cuidado à saúde. Rio de Janeiro: UERJ/ IM S/ABRASCO; 2001. p. 39-64.

11. Lück H. Pedagogia interdisciplinar: fundamentos teórico-metodológicos. Petrópolis: Vozes; 2002.

12. Feuerwerker LCM, Sena RR. Interdisciplinaridade, trabalho multiprofissional e em equipe. Sinônimos? Como se relacionam e o que têm a ver com a nossa vida? O Iho Mágico 1998; 5(18):5-6.

13. Campos GW. Subjetividade e administração de pessoal: considerações sobre modos de gerenciar a trabalho em equipes de saúde. In: M erhy EE, Onocko R, organizadores. Agir em saúde: um desafio para o público. São Paulo: Hucitec; 1997.

14. Vasconcelos EM. Complexidade e pesquisa interdisciplinar: epistemologia e metodologia operativa. Petrópolis: Vozes; 2002. p. 343.

15. Who Study Group On Multiprofessional Education of Health Personel: The Team Approach. Learning together to work together for health. Geneva: World H eath Organization; 1988.

16. Garcia MAA. Knowledge, action and education: teaching and learning at healthcare centers. Interface 2001; 5(8):89-100.

17. Bordenave JED. La transferência de tecnologia apropiada al pequeño agricultor. Revista Interamericana de Educação de Adultos 1983; 3:1-2.

18. Fujimura I. Apresentação. In: M arcondes E, Gonçalves EL, organizadores. Educação médica. São Paulo: Savier; 1998. p. 245-247.

Artigo apresentado em 23/09/2005

Aprovado em 08/06/2006

Versão final apresentada em 18/08/2008 